\title{
Convivencia escolar. Razones para la polémica de un problema actual
}

\author{
Shool life. Reasons for the controversy of a current problems
}

Jorge Alexander Hernández Benítez ${ }^{\natural}$ (iD Regina De La Caridad Agramonte Rosell²

Eduardo Menéndez Alvarez ${ }^{3}$

${ }^{1 .}$ Universidad Metropolitana de Ciencia Y Tecnología. Ciudad de Panamá, Panamá
${ }^{2}$ Universidad Científica del Sur. Lima, Perú
${ }^{3 .}$ Universidad Le Cordon Bleu. Lima, Perú

Recibido: 05/10/2021 Revisado: 20/09/2021_Aceptado: 06/08/2021 Publicado: 31/10/2021

\section{RESUMEN}

En el presente texto se analizan los términos convivencia escolar y estrategia pedagógica desde sus perspectivas, semánticas, epistemológicas, legales y conceptuales; basados en los cuatro aprendizajes fundamentales considerados como los pilares del saber universal; de igual forma, se toma en cuenta el impacto que estos tienen en las relaciones humanas e interpersonales en el contexto escolar, y de cómo pueden ser usados a modo referentes para el desarrollo de programas, manuales y demás documentos que conforman los marcos normativos que rigen la escolaridad y otros ambientes educativos; todo lo anterior es tomado a consideración con el objetivo de indagar sobre de los orígenes, evoluciones, implicaciones y posibles soluciones de los conflictos que a diario se presentan en las escuelas colombianas. El documento también analiza, el componente democrático y en qué medida su adaptación interactúa con la convivencia escolar y su implementación en el campo educativo. Además, para fundamentar el texto, se escogen algunos conceptos expuestos por diversos autores y se hace una relación analítica de la pertinencia de estos hacia el proceso investigativo, partiendo de los contextos en donde se dieran lugar a realizar dichas iniciativas, de esa forma generar expectativas e ideas para el desarrollo de proyectos encaminados a la mediación de las relaciones personales en pro del mejoramiento del ambiente escolar y generación de espacios, democráticos, incluyentes y de pensamiento. Palabras clave: Convivencia escolar, estrategia pedagógica, convivencia democrática, clima escolar.

\begin{abstract}
In this text, the words school coexistence and pedagogical strategy are analyzed from their, semantics, epistemological, legal and conceptual perspectives; based on the four fundamental learnings which are considered as the pillars of the universal knowledge; Similarly, the impact


they have on human and interpersonal relationships in the school context is taken into account, and how they can be used as references for the development of programs, manuals and other documents that make up the normative frameworks that rule schooling and other educational environments; all of the above is taken into consideration in order to inquire about the origins, evolutions, implications and possible solutions of the conflicts that nowadays occur in Colombian schools. The document also analyzes the democratic component and to what extent its adaptation interacts with school coexistence and its implementation in the educational field. In addition, to substantiate the text, some concepts exposed by various authors are chosen and an analytical relationship is made of their relevance to the investigative process, starting from the contexts in which these initiatives would take place, thus generating expectations and ideas for the development of projects aimed at the mediation of personal relationships in favor of the improvement of the school environment and the generation of spaces, democratic, inclusive and of thought. Keywords: School coexistence, pedagogical strategy, democratic coexistence, school environment.

\section{INTRODUCCIÓN}

La sociedad humana se desarrolla en un contexto de comunicación permanente; los conflictos por lo general trascienden ante las complejidades de la convivencia. Lo anterior se puede vivenciar en espacios laborales, familiares, escolares; pues donde quiera que no se cuiden las relaciones interpersonales habrá un escenario para conflictos, que no siempre resultan fáciles de resolver. Y es que la etapa actual se caracteriza por una serie de contradicciones donde la incertidumbre y la globalización de la vida cotidiana, aceleran los procesos, se introducen nuevos códigos, emergen insatisfacciones y con ello serias dificultades para conciliar y lograr entendimiento en espacios privados y colectivos de paz. En apoyo de esta idea, se considera pertinente lo postulado por Pérez I. (2016), acerca de que "la democracia tiene que ser el marco dialógico que nos permita conocernos y convivir con la diferencia hasta enriquecernos mutuamente". De modo que, como puede apreciarse, en algunas instituciones educativas de Colombia, sus entornos escolares y relaciones intersubjetivas evidencian notorias carencias en cuanto al respeto, tolerancia, amor, solidaridad, entre otros valores que constituyen la base de un comportamiento adecuado. A raíz de ello, los estudiantes adoptan conductas violentas que muchas veces son inaceptables, afectando indirectamente su formación integral y por ende la de los demás; hasta llegar, en algunos casos, a resultados fatales para las víctimas de tales comportamientos. Por tal motivo, es imperioso que los docentes desempeñen un rol fundamental en la vida de cada uno de los estudiantes y miembros de la comunidad educativa, buscando métodos, técnicas, o cualquier clase estrategias que les brinden una perspectiva adecuada sobre su visión del mundo para así formar personas virtuosas dentro de la sociedad. En el trabajo académico de Campdesuñer (2020), titulado: Plan de mejora y convivencia democrática en interculturalidad. Un estudio en establecimientos educacionales de la comuna de Santiago de Chile, se sostiene que:

En las últimas décadas el tema de la convivencia escolar ha cobrado mucha importancia en el contexto educativo. La cada vez más colorida y diversa fisionomía de las escuelas, así como también el heterogéneo entramado social y 
cultural de los estudiantes, han ido de la mano con la presencia de la violencia y el racismo como expresiones de exclusión en el ámbito escolar (p. 87).

Con sustento en esta idea, se explica la importancia de la conceptualización de los términos "convivencia escolar y estrategias pedagógicas", tomando como referentes una amplia representación de autores estudiosos del tema.

La convivencia escolar resulta ser un tema polémico dadas las complejidades de las relaciones interpersonales a nivel global. Lo cual explica una amplia presencia de información en revistas indexadas de alto impacto y libros especializados (Campdesuñer, 2015); lo que puede interpretarse como una muestra del interés que la comunidad académica y científica internacional le otorga, a partir de las contradicciones en torno a esta episteme; de lo anterior, se deriva la necesidad de que el informe escrito de esta tesis, presente un acercamiento a la conceptualización de nociones básicas, respaldadas con algunos estudios (tesis, artículos, ensayos, normatividad, teorías) relacionados con el tema, dada su implicación lógica, metodológica y epistemológica. Desde una perspectiva teórica, para la convivencia escolar existe cierto consenso en su definición, es decir que hay una estandarización en su descripción. Dentro de la acción de vivir, la educación estructura cuatro aprendizajes fundamentales, dándole sentido al conocimiento adquirido, no solo en las aulas de clases, sino también en otros contextos. Estos aprendizajes serán los pilares del saber: el primero es el aprender a conocer, para comprender el mundo; continuando con el aprender a hacer, para transformar de manera positiva en contexto; siguiendo con el aprender a vivir juntos, para fortalecer las relaciones interper- sonales y la ayuda hacia los demás; para terminar en el último pilar, aprender a ser, que es la fusión de los anteriores. De ahí que la convivencia escolar haga referencia al conjunto de relaciones que ocurren entre las personas que hacen parte de la comunidad educativa que debe enfocarse en el logro de los objetivos educativos y su desarrollo integral.

En esta misma línea, aparece Mockus afirmando que La convivencia escolar resume el ideal de la vida en común entre las personas que forman parte de la comunidad educativa, partiendo del deseo de vivir juntos de manera viable y deseable a pesar de la diversidad de orígenes. Así mismo, esta se relaciona con construir y acatar normas; contar con mecanismos de autorregulación social y sistemas que velen por su cumplimiento; respetar las diferencias; aprender a celebrar, cumplir y reparar acuerdos, y construir relaciones de confianza entre las personas de la comunidad educativa (Mockus, 2002); haciendo referencia a lo anterior, se considera oportuno el planteamiento de Antanas Mockus, autor que, por su trayectoria académica y experiencia laboral, es de considerarse un especialista de aportaciones trascendentales en este tema. No obstante, también es de considerar que, al momento de especificar el rol de la escuela en el proceso de formación para la convivencia, el autor define incipientemente su postulado, ya que la formación de conductas, pensamientos y habilidades se deben fortalecer es en la escuela, de tal manera que un niño o joven estudiante no puede dejarse que lo realice de forma empírica o voluntaria (Pediatrics, 2018). En virtud de lo cual, el texto referenciado no ofrece la suficiente ilustración en cuanto a la función de la escuela en la formación infantil, en el tema de estudio. Ahora bien, la reflexión sobre la convivencia 
escolar trae consigo la necesidad de comprender esta noción desde su carácter procesal, flexible, continua; que debe ser construida cada día en las instituciones educativas desde donde se fomenta una interacción de conductas, formas de pensamiento, experiencias, visiones del mundo, entre otros factores que contribuyan al desarrollo de ambientes escolares democráticos, en los cuales la participación y el fomento de identidad desde la diferencia, sean centrales (Pérez R. , 2007). Es decir que, no solamente la escuela debe verse como el sitio para aprender normas de convivencia y todo tipo de conductas sociales, es también el sitio para poner en práctica todo lo aprendido: la democracia, el respeto, la inclusión y todas las demás condiciones que hagan tomar conciencia de las diferencias que existen en cualquier nicho social.

De otro lado, autores como (Fierro y Carbajal (2015), profundizan en el estudio de las características de la convivencia escolar, estableciendo algunas de las perspectivas necesarias en el desarrollo de análisis e investigaciones atinentes a la temática enunciada. Así, la primera determinación a ser considerada es el estudio de clima escolar, de la violencia y/o de su prevención; lo anterior, con el fin de identificar patrones de conducta y factores de riesgo que delimiten la alteración del ambiente escolar; para, de esa forma, continuar con el proceso de augurar las emociones de los sujetos (estudiantes), estructurando la convivencia en un estado de educación "Socio-Emocional", que centra su atención al desarrollo de habilidades sociales como mecanismo principal de la convivencia y por tanto como componente regulador en la mejora de las relaciones interpersonales en el contexto educativo (Fierro y Carbajal, 2015). Por último, se debe basar en los principios democrá- ticos y comunitarios, que enmarcan las pautas de civismo que, desde la escuela, deben concebirse; de tal modo que la convivencia sea un elemento de educación para la ciudadanía y la democracia, que se aplique para considerar los procesos cotidianos de participación como oportunidades para desarrollar capacidades cívicas, entre las cuales se logre aprender a reconocer la diversidad de identidades y capacidades de otras personas, valorar la pluralidad de ideas, entre otros. Estas perspectivas, son herramientas imprescindibles para tener en cuenta - en las escuelas y centros de educación y/o formación - en el desarrollo de manuales de convivencia, normas, reglamentos, etc.; ya que apuntan a elementos importantes como la democracia, el civismo, las emociones y el ambiente escolar; siendo estos, referencias de gran aporte a la consolidación estructural de la tesis actual. Es necesario destacar lo expuesto sobre la Educación para la Ciudadanía y la Democracia, debido a que se basa en la formación para el respeto de la diversidad, la tolerancia, participación etc.

Del mismo modo, al momento de mencionar las características que se desprenden de la convivencia escolar, se genera una arista denominada "clima escolar" que algunas veces tienden a confundirse. Para (Arón \& Milicic, 1999), existe una división o discrimen del señalado concepto, y argumentan que los climas escolares pueden clasificarse en nutritivos y tóxicos; los primeros brindan al conglomerado la impresión de motivación al participar en actividades de la escuela, generando una convivencia positiva, en la cual existe interés por el aprendizaje, respeto entre los profesores y alumnos, confianza, etc. Asimismo, permite un ambiente físico adecuado para las actividades escolares cotidianas, reconocimiento y valoración de críticas, castigos y 
realización de actividades divertidas. Por el contrario, los climas escolares "tóxicos" se caracterizan por evidenciar conductas contrarias a las del clima nutritivo, los que derivan en conflictos no favorables para los procesos académicos y formativos entre los miembros de la comunidad educativa. Esta clasificación podría ser considerada como polarizada, ya que enmarca drásticamente la definición de clima escolar, sin dar espacio a un término medio para el concepto. En la definición de clima nutritivo, se describe un contexto poco probable; que, al aterrizarlo a la práctica, se nota un amplio margen con respecto a la realidad, debido a que, aunque las condiciones óptimas estén dadas, siempre habrá inconvenientes, puesto que la conducta humana varía de acuerdo a cada individuo (Peña, Sánchez y Menjura, 2015). Para sintetizar, un tercer clima escolar de término intermedio generaría una discusión más acertada, entraría a ser denominado como clima "ecléctico".

La importancia que viene teniendo la convivencia escolar, desde hace más de dos décadas, en la normatividad e investigación científica, en factores como el desarrollo conductual en educación primaria, asientan su base en la integración del contexto escolar por medio de la regulación de elementos; cuando este proceso adquiere matices positivos o negativos, los factores conductuales que componen la convivencia y los procedimientos regulares de la escuela se ven afectados puesto que se altera el orden. No obstante, no debe limitarse al desarrollo de la personalidad solamente en los niveles de educación primaria; ya que estos conflictos escolares, se ven de manera más evidente y recurrente en la educación secundaria, sobre todo en los grados $7^{\circ}, 8^{\circ}$ y $9^{\circ}$. Esto se debe quizás por el cambio a nivel fisiológico al que generalmen- te se encuentran los estudiantes al cursar los mencionados grados (Lozano, 2019). No es un detalle menor, dejar la secundaria por fuera de la teoría. Muchos niños o adolescentes no han formado su carácter y moldearlos es papel de la escuela, por ende, en esta etapa de sus vidas en cuando resulta más relevante hacer de ellos ciudadanos democráticos y pacíficos.

En este punto, es importante resaltar que, en el estudio sobre las definiciones de convivencia escolar, surge una integración de conceptos relacionados con la democracia, para hacer una concepción metodológica de su percepción. Por ello, se supone que las escuelas desarrollen procesos por medio de los cuales los estudiantes adquieran la habilidad para regular sus emociones y sentimientos, que se reflejen en formas de manejo de conflictos en las que prevalezca el consenso y el dialogo; luego, es preciso contar en el trabajo de aula con herramientas como la deliberación, el debate, así como la participación en la formulación y seguimiento de normas tanto en el aula como fuera de ella; se resume entonces que la convivencia democrática necesita fomentar el valor de solidaridad, puesto que existe un sesgo social que indica que no todos tienen la oportunidad de expresarse y que sus opiniones sean tomadas en cuenta, para la toma de decisiones.

Esto lleva a destacar el fomento de la empatía y la adquisición de perspectiva, que facilite obtener consensos en puntos de opiniones diferentes a las propias, para identificar y mediar conflictos. Hasta este punto, los autores coinciden con la búsqueda teórica de la presente tesis, pero las experiencias vividas en entornos educativos, muestran que no toda escuela tiene como política institucional, las herramientas legales, físicas o humanas, para sobrellevar situaciones que alteren la norma- 
lidad pedagógica; luego entonces, esta teoría plantea un postulado que no está en la posición de ser adoptado por un sistema educativo complejo, o de grandes números de escuelas.

También se halló, desde otra concepción teórica formulada por Yamanija, Omura, y Barrientos (2018), que, "la convivencia democrática se basa en la práctica de relaciones interpersonales que se modulan y permiten vivir en sociedad". Es decir, que este concepto va más allá de los contextos escolares; la interiorización de él, va a ser factor determinante en el desarrollo social, partiendo de las conductas y formas de interacción comunitaria. De ahí la importancia de poseer herramientas, humanas, legales y sociales, en el organigrama educativo de la escuela, pensando en el hombre del mañana y en el "producto" final que se convertirán los estudiantes al enfrentarse a la sociedad y las diversas situaciones que devienen del diario acontecer (Yamanija, Omura, y Barrientos, 2018).

Por otro lado, se hace casi que obligatorio mencionar la inclusión, ya que es un tema de mucho auge y relevancia en la elaboración de diseños curriculares, planes de estudio, normatividad escolar o cualquier tipo de planeación, tanto interna, como a nivel gubernamental. Lo anterior vislumbra la forma en cómo la escuela se estructura pedagógicamente y establece sus políticas enfocadas a mantener la asistencia de todos los estudiantes. De tal manera que la convivencia inclusiva requiere de factores en su implementación, en los cuales deben existir en su estructuración elementos que atiendan las necesidades de personas con disposiciones físicas y/o intelectuales diferentes, así como personal capacitado para atender las diversidades (de géne- ro, cultura, religión, lengua, condición física etc.); que fomenten la participación y sentido de pertenencia de los miembros de la comunidad educativa y el trabajo colaborativo.

Entonces, estas características, formulan algunos criterios sustanciales a tener en cuenta al momento de formular estrategias pedagógicas en trabajos de investigación, por lo que se hace importante tener definidas las particularidades de cada contexto, para proceder a la aplicación de un elemento documental en pro de la convivencia escolar.

En esta instancia, y haciendo referencia al marco legal, se puede notar que el amparo jurídico de todo concepto pedagógico e investigativo, debe servir como fundamento legal, para el amparo ante la Ley; en Colombia existe un conjunto de normas que regulan el funcionamiento escolar a nivel general y también existen normas, que respaldan la convivencia escolar directamente; es este caso el Sistema educativo colombiano está regulado por la Ley 1620 de 2013 (MEN, 2013), normatividad educativa constituida por artículos que enmarcan las conductas atípicas que alteran el normal desarrollo del proceso académico de las escuelas nacionales. La implementación y difusión de esta ley, comenzó desde el año 2013 en todas las escuelas nacionales, tanto públicas como privadas, pero al día de hoy, se refleja una falta de consolidación y aprehensión por parte de la comunidad educativa, en cuanto a sus objetivos, por el hecho en que, en los últimos años, muchos casos manifiestos han ido en crecimiento en nuestro país en términos de violencia sexual, embarazo precoz, acoso escolar y transmisión de enfermedades de trasmisión sexual. 
Esta falencia institucional, no se manifiesta estrictamente por fallas en las instituciones educativas, dado que, de cierta forma, aquí la mayor responsabilidad la debe asumir el propio el Ministerio de Educación Nacional (MEN), órgano que no ha implementado políticas precisas para difundir la esencia de la ley, ni tampoco se ha dispuesto un plan de trabajo organizado para capacitar al cuerpo docente (docentes, de aula, coordinadores, psicólogos, rectores) en el manejo tanto de la norma, como del debido proceso de las situaciones de alteración de la convivencia que se presentaren en las escuelas. Este referente muestra señales de la realidad en que se encuentra el manejo de la convivencia infantil y de adolescentes en el país; y de la notoria incompetencia por parte de los entes de control para poner en práctica esta importante ley, y de esa forma, garantizar la integridad de la niñez y juventud nacional que se ha visto afectada por diversidad de factores, entre ellos, la convivencia escolar.

La convivencia escolar no es ajena a las percepciones y teorías epistémicas de la educación y la piscología. Así, el aprendizaje significativo postulado por David Ausubel (1997), es muy pertinente en el estudio de los aspectos y factores conductuales de los jóvenes, dado que, al momento respetar normas y formar el carácter de la personalidad, la fundamentación de lo aprendido y la experiencia por medio de la cual el conocimiento fue adquirido, cumplen un rol indispensable en los esquemas de pensamiento del estudiante. De hecho, las concepciones epistémicas de Ausubel se presentan como componentes de colaboración para la convivencia escolar, integrando la comunicación asertiva, la participación de los actores educativos, la asesoría escolar y la interdisciplinariedad.

También se tiene que, en la construcción de la teoría del conocimiento, el pragmatismo visto como actitud, fomenta la apropiación adecuada de un carácter de ideas en pro de la sana convivencia; pero debe ser analizado desde la aplicación de la coerción a los que, de una u otra forma, infrinjan las normas, en cuanto a que en esta se buscar es mejorar a través de la toma de conciencia, mas no de elementos punitivos y de limitación de derechos.

Para Reutert y Castro (2019), "la convivencia escolar es una construcción colectiva y dinámica que surge a partir de las diferentes interacciones al interior de un establecimiento educacional". Así mismo los postulados del teórico constructivista Jean Piaget (1996), aseveran que, por medio de la interacción entre las personas, se va creando y fomentando la convivencia escolar, de modo que los educadores tienen la oportunidad de encaminar a los estudiantes para formarles de manera sana, productiva y para el bien común de su sociedad.

Además, el constructivismo como fuente de creación de relaciones interpersonales, posee características que lo conectan con el factor de convivencia, como se comprende en los estudios de Reutert y Castro (2019). Estas relaciones se enfocan al afecto en el trato interpersonal, para promover las posibilidades que tienen los diferentes miembros de participar y expresarse. La escuela tiene como deber brindar espacios para actividades diversas de los alumnos, que se concentren en la actitud que tomen los estudiantes cuando sientan la acogida o rechazo, la formas y el criterio que se adopta para distribuir el 
poder, los estilos de trabajo entre profesores.

La integración de todos los factores que se referencian, hace que se cree la constricción de convivencia social, en este caso, de un tipo de convivencia en el contexto escolar. Y esta fuente nos brinda un carácter epistemológico para el desarrollo de investigaciones y trabajos científicos. Existen fundamentos epistémicos que consolidan la convivencia escolar, porque se parte de que la interacción entre seres humanos es compleja, por la heterogeneidad de estos debido a sus diferencias religiosas, políticas, culturales, entre otras, factor que no es solo evidenciado en la escuela, sino también en el contexto familiar; sin embargo, una vez que se ingresa al sistema educativo, el estudiante deberá aprender desde otro contexto, otras diferencias y aceptarlas como parte de su formación social.

En términos de la educación, la convivencia y el constructivismo se integran por medio de la promoción del ser humano como sujeto, miembro de una sociedad regida por normas de conducta, reflejadas en deberes y derechos; a medida que se adapte al medio ambiente y social de manera correcta. Aunado a ello, la educación proporciona los medios para fortalecer la formación del individuo en concordancia con los requerimientos de la realidad social que lo contextualiza, como lo representa (Piaget, 1996), al afirmar que, "educar es forjar individuos capaces de una autonomía intelectual y moral que respeten esa autonomía y la del prójimo, en virtud de la regla de la reciprocidad" (p.56); es decir que, la educación promueve siempre no solo la intelectualidad, si no la vida social.
De una manera más explícita, la teoría sociocultural de Vygotsky (1979), tiene su cimiento en el aprendizaje sociocultural del individuo y así mismo en el entorno en el que se desenvuelve, es decir que el individuo tiene la habilidad de aprender por medio de la observación de la conducta de otros; Vygotsky expresa que el ser humano es dinámico y se adapta a su entorno del cual aprende y pone en práctica a través de su conducta. Esta teoría también expone que en el desarrollo del conocimiento se da que la relación entre la persona y su entorno es un principio básico; luego se aprende con la experiencia. En el constructivismo de Vygotsky los docentes y padres son mediadores de la relación y reacción del niño con el mundo, esto es base fundamental para el desarrollo de las diferentes investigaciones, ya que, por medio de la elaboración de una implementación pedagógica, las opiniones y aquiescencia de los padres de familia es factor determinante en el éxito de dicha implementación (Vygotsky, 1979). Por otra parte, una de las teorías de Seymour, (Ortega, 2015) señala que "los estudiantes deben ser estimulados, para el aprendizaje de normas, sensaciones cenestésicas y propioceptivas, que ayudan a la realización de actividades, y así representar los esquemas de su entorno" (p.6). Aquí en enfoque analítico y del conjunto de códigos, establecen la relación íntima entre el enfoque de Seymour y la convivencia escolar, ya que las normas, son códigos conductuales, que deben ser respetados.

Las estrategias pedagógicas, se han convertido en una de las herramientas más eficaces para el desarrollo de planteamientos y resoluciones de conflictos relacionados 
con los aspectos educativos, en este caso los que tienen que ver con la convivencia escolar y democrática. En el contexto educativo, el término estrategia es de uso frecuente en todo tipo de documentos inherentes al quehacer escolar, y también es frecuente encontrarlo asociado con la pedagogía, principalmente, en la tesis que hoy nos ocupa, ya que ambos son aunados con el propósito de creación, desarrollo y culminación de innumerables propuestas de investigación. Para representar un bagaje amplio de estos dos términos, a continuación, se exponen algunas definiciones de autores e investigadores, en pro de fortalecer el marco teórico de este proyecto.

De acuerdo al diccionario de la Real Academia Española, "estrategia, se define como el arte de coordinar acciones y de maniobrar para lograr un objetivo o un proceso; que se puede demostrar a través de habilidad, talento, destreza, disposición, creatividad, inspiración, disciplina, técnica para hacer algo" (RAE, 2013). Pero de esta expedita definición, que da la RAE, se devienen ciertas características, que muchos autores describen, teniendo en cuenta la funcionalidad de esta, y su aplicación a los diversos tópicos en que sea usada, para el caso que nos concierne, se marcan las principales particularidades en el campo de la educación. Cuando las estrategias son consideradas alternativas para el desarrollo de investigaciones y fundamentación de teorías en la educación superior y posgradual, se toman como modelos para el mejoramiento del desempeño profesional pedagógico de los docentes; en tal virtud, una estrategia pedagógica se define como un programa de superación para el mejoramiento de los procesos investigativos de carácter universitario, en esta descripción la estrategia pedagógica, se considera un proyecto que busca el beneficio de una determinada comunidad y más allá.

La estrategia pedagógica en términos de amplitud y representación a gran escala, contiene características muy expresas, al momento de ser aplicada en el desarrollo de investigaciones. En tal sentido, Sierra (2007) hace un compendio de dichas características, precisando que, la estrategia está sujeta a ser cambiada, dada con exactitud, concreta a partir de las modificaciones a que diera lugar. La estrategia pedagógica, en cuanto al rol del docente, debe dar solución a los objetivos establecidos en el currículo, conformado por los niveles de educación.

En el haber de la educación han existido prácticas pedagógicas tradicionales, que conlleva un gran espectro de actividades dentro de las cuales la más conocida es la denominada clase magistral; la aplicación de este modelo de clases centra sus objetivos en la adquisición de conocimiento, por medio del habla, donde los alumnos son solo receptores. Las clases magistrales, tienen una consecuencia positiva, cuando el estudiante buscaba adquirir el conocimiento de forma unilateral, aupado por su motivación intrínseca compuesta por el docente y los contenidos que pretendía enseñar; la actualidad ha hecho cambiar de manera amplia esta concepción, y ha hecho que la jerarquización de las clases magistrales, en la que el docente era el centro de la enseñanza; se haya modificado y tanto el docente como el grupo de estudiantes, se encuentran en el mismo nivel y se busca amalgamar los objetivos del docente con los de los estudiantes.

Con el fin de que las estrategias no queden relegadas a simples técnicas y recetas, 
sino que sean aplicadas pragmáticamente en el proceso de enseñanza - aprendizaje, y de esa forma tener en cuenta una un propósito pedagógico; y no solo sea limitada a las acciones tradicionalistas de las clases, que poco fomenta la imaginación y creatividad de los estudiantes.

De modo que, para finalizar, es de señalar que, en Pedagogía, utilizar una estrategia, ayuda a establecer la dirección inteligente, y desde un punto de vista amplia y global, también son útiles para las acciones encaminadas a resolver los problemas que se presenten en el trasegar del ejercicio de la cotidianidad. El desarrollo de la personalidad del estudiante, como individuo resolutivo de problemas, está sujeta al direccionamiento del proceso pedagógico, en el que se pretende cambiar un estado de conducta y de pensamiento insipiente a uno con mayor grado de saberes y que tenga la habilidad de ponerlos en práctica, para el bien propio y el general.

\section{CONCLUSIONES}

Se señala la importancia de resaltar las aportaciones de los autores incluidos en este estudio, dada su estrecha relación de sus formulaciones y resultados investigativos, los que brindan elementos de apoyo a la fundamentación teórico-conceptual sobre le temática aquí abordada, para el desarrollo de procesos investigativos y científicos concernientes.

Por medio de la conceptualización de los términos "convivencia escolar" y "estra- tegias pedagógicas" se enriquecen las ideas del investigador y pueden formar un criterio propio para poner en práctica en sus proyectos o promover los de otros, puesto que con lo visto se fortalecen las perspectivas de sus ideas y les permiten interactuar con estas.

El acervo documental referenciado, es un repertorio amplio en cada una de las temáticas conceptualizadas sobre convivencia escolar y estrategias para su mejoramiento; ayudando al investigador analizar minuciosamente lo expuesto por cada uno de los autores citados. Por consiguiente, es pertinente que en el interior de la documentación contenida es este texto, se identifique los elementos práctico-pedagógicos requeridos en la implementación de una estrategia pedagógica; partiendo de la definición de las características intrínsecas de la convivencia escolar y cada una de sus particularidades.

La educación se encuentra frente a un desafío, que se basa en una nueva travesía académica enfocada en la generación de nuevos y actualizados modelos pedagógicos que sean los derroteros de un proceso académico proactivo, que se dirija hacia el fomento de habilidades críticas, creativas a los educandos, en todos los niveles de aprendizaje. Pues coincidimos con lo postulado por Berkel y Schmidt (2011), en el sentido en que, para lograr este tipo de paradigmas educativos, es menester enriquecer las capacidades creativas, investigativas de los docentes en el aula y por fuera de ellas.

\section{REFERENCIAS BIBLIOGRÁFICAS}

Aguilar, P. (2018). Contextos que matan. Loyola \& Psico. 
Algara, A. (2016). Los acuerdos del aula una estrategia de convivencia para fortalecer la democracia en la escuela primaria. Revista Ra Ximhai, 12(3 Especial). https://drive.google.com/file/d/0B_QQ0W8TI5accXhEeUd2MVJwVm8/view

Arón, A., y Milicic, N. (1999). Clima social escolar y desarrollo personal. Agencia Nacional de Investigación y Desarrollo -ANID. https://doi.org/10.2307/j.ctt1trkk9z

Ausubel, D. (1997). Psicología Educativa: un punto de vista cognitivo. Trillas.

Benites, L. (2011). Convivencia escolar y calidad educativa. Cultura 25, pp. 146-158. https://www.revistacultura.com.pe/revistas/RCU_25_1_convivencia-escolar-y-calidad-educativa.pdf

Berkel, A., y Schmidt, M. (2011). The role of lectures in problem-based learning. ERIC. https://doi.org/?id=ED453283

Campdesuñer, L. (2015). Apuntes para una comprensión dinámica. Foro Educacional.

Campdesuñer, L. (2020). Plan de mejora y convivencia democrática en interculturalidad. Un estudio en establecimientos educacionales de la comuna de Santiago de Chile. Universidad de Sevilla. https://hdl.handle.net/11441/102381

Campos, A. (2012). Metodología para la enseñanza del Derecho del Trabajo y de la Seguridad Social en el Espacio Europeo de Educación Superior. https://burjcdigital.urjc.es/bitstream/handle/10115/12618/Dialnet-Metodologia Para LaEnsenanzaDelDerechoDelTrabajoYDeL-3866238.pdf?sequence=1\&isAllowed=y

Carrasco, C., y Trianes, M. (2010). Clima social, prosocialidad y violencia como predictores de inadaptación escolar en primaria. European Journal of Education and Psychology. https://www.redalyc.org/pdf/1293/129315468007.pdf

Casas, J., y Ortega, R. (2016). Valoración del alumnado de primaria sobre convivencia escolar: El valor de la red de iguales. Psicoperspectivas, 21. http://dx.doi.org/10.5027/psicoperspectivas-Vol15-Issue2-fulltext-760

Casas, J., y Ortega, R. (2017). Desarrollo y validación de la Escala de Convivencia escolar (ECE). Universidad de Sevilla. http://dx.doi.org/10.11144/Javeriana.upsyl6-1.dvec

CERE (1993). Evaluar el contexto educativo. Vitoria: Ministerio de Educación y Cultura, GobiernoVasco.http://www.opech.cl/bibliografico/Participacion_Cultura_Escolar/Clima Escolar_Cornejo_Redondo.pdf 
Chaparro, A. y Caso, J. (2015). Desarrollo de un instrumento de evaluación basado en indicadores de convivencia escolar democrática, inclusiva y pacífica. Perfiles educativos, 3. https://www.redalyc.org/articulo.oa?id=13239889002

Del Rey, R. y Ortega, R. (2004). Resistencias, conflictos y dificultades de la convivencia. Edebé. http://dx.doi.org/slideplayer.es/slide/3102099/

Díaz, P., y Alea, M. (2015). http://dx.doi.org/ Revista habanera de ciencias médicas. Doi: $10.5027 /$ psicoperspectivas-Vol18-Issuel-fulltext-1486

Fazio, M., y Fernández, F. (2004). Historia de la Filosofía contemporánea. Palabra.

Fierro, C., y Carbajal, P. (2015). Convivencia escolar: Una revisión del concepto. Universidad Iberoamericana de León. Doi: 10.5027/psicoperspectivas-Vol18-Issuel-fulltext-1486

Fierro Evans, María Cecilia. (2013). Convivencia inclusiva y democrática: Una perspectiva para gestionar la seguridad escolar. Sinéctica, (40), 01-18. Recuperado en 15 de octubre de 2021, de ht t p://www.scielo.org.mx/scielo.php?script=sci_arttext\&pi$\mathrm{d}=$ S1665-109X2013000100005\&lng=es\&tlng=es.

Fierro, M. y Carbajal, P. (2010). Ojos que sí ven: Casos para reflexionar sobre la convivencia en la escuela. SM. https://www.researchgate.net/publication/49111329

Flórez, A. y Segura, Ó. (2013). Análisis del fenómeno bullyng en los adolescentes del $5^{\circ}$ y $6^{\circ}$ año de la escuela primaria Miguel Hidalgo y Costilla, turno matutino del ciclo escolar 2012-2013, en San Fernando Huixquilucan, Estado de México. Universidad Autónoma del Estado de México -UAEM. http://ri.uaemex.mx/handle/20.500.11799/13843

Furman, W. (2003). The role of romantic relationships in adolescent development. ResearchGate, 4. DOI:10.1017/CBO9780511551963.016

Golleman, D. (1995). Inteligencia emocional. Kairós.

Govinda, R. (2009). Towards inclusive schools and enhanced learning. UNESCO.

Guédez, V. (2005). La diversidad y la inclusión: Implicaciones para la Cultura y la Educación. Sapiens.revista universitaria de investigación. 6 (1). 107-132. https://www.redalyc.org/articulo.oa?id=41060107 
Isaza, A. (2005). Clases magistrales versus actividades participativas en el pregrado de medicina: de la teoría a la evidencia. Revista de Estudios Sociales, (20), 83-91. ht t p://www.scielo.org.co/scielo.php?script=sci_arttext\&pi$\mathrm{d}=\mathrm{S} 0123-885 \mathrm{X} 2005000100006 \& \operatorname{lng}=$ en\&tlng=es.

Lanfrancesco, G. (2003). La educación integral en el preescolar. Magisterio.

León, F. (2014). Teoría del conocimiento. Valencia, Venezuela: Universidad de Carabobo.

Lozano, S. (2019). Por qué debes estar alerta a los cambios de conducta de los niños y adolescentes. La Vanguardia.

Ludeke, R. J., y Hartup, W. W. (1983). Teaching behaviors of 9- and 11-year-old girls in mixed-age and same-age dyads. Journal of Educational Psychology, 75(6), 908-914. https://doi.org/10.1037/0022-0663.75.6.908

Martínez, L. (2017). Análisis del Sistema Nacional de Convivencia Escolar y Formación para el Ejercicio de los Derechos Humanos, Sexuales y reproductivos desde la perspectiva de la eficacia simbólica. Pensamiento Jurídico N49, 147. file://C:/Users/USUARIO/Downloads/59377-Texto\%20del\%20art\%C3\%ADculo-446159-1-10-20191118.pdf

MEN (2013). Ley 1620 de 2013: "Por la cual se reglamenta el Sistema Nacional de Convivencia Escolar y formación para el ejercicio de los Derechos Humanos, la Educación para la Sexualidad y la Prevención y Mitigación de la Violencia Escolar". Imprenta Nacional. https://www.mineducacion.gov.co/1759/articles-327397_archivo_pdf_proyecto_decreto. pdf

Méndez, V. y Cartín, J. (2012). Los modelos pedagógicos centrados en el estudiante: apuntes sobre los procesos de aprendizaje y enseñanza. Proifed. file://C:/Users/USUARIO/ Downloads/Mendez_Villalobos_Dalton_Cartin_Riedra_Modelos_Pedagogicos_Centra. pdf

Mintzberg, H. (1995). Destreza en la estrategia. Biblioteca de planeación estratégica. https:// andrader0.tripod.com/docs/paradigmas/las5ps.pdf

Mintzberg, H. y Quinn, J. (1995). El Proceso Estratégico. https://www.academia.edu/10869386/AE_El_Proceso_Estrategico?from=cover_page

Mockus, A. (2002). Convivencia como armonización de ley, moral y cultural. Educación para aprender a vivir juntos, 14. http://www.ibe.unesco.org/fileadmin/user_upload/archive/ Publications/Prospects/ProspectsPdf/121s/121smock.pdf 
Monereo, C. (1995). De los procedimientos a las estrategias: implicaciones para el Proyecto Curricular Investigación y Renovación escolar. IRES. https://dialnet.unirioja.es/servlet/articulo?codigo $=116921$

Morín, É. (1990). Introducción al Pensamiento Complejo. Gedisa. https://d1wqtxts1xzle7.cloudfront.net/54111654/LIBRO-introduccion_al_pensamiento_ complejo_Morin-with-cover-page

Moya, M. y Hernández, J. (2009). Un estilo de aprendizaje, una actividad. Diseño de un plan de trabajo para cada estilo. Estilos de Aprendizaje. https://revista.ieee.es/index.php/estilosdeaprendizaje/article/view/895

Muñoz, C. (2019). Análisis situacional de los manuales de convivencia escolar en las instituciones educativas distritales de la localidad San Cristóbal de Bogotá D.C. ORATORES, 17. https://revistas.umecit.edu.pa/index.php/oratores/article/view/281

Ortega, B. (2015). Convivencia pacífica en niños y adolescentes. UNPN. D.F. http://repositorio.ug.edu.ec/handle/redug/30430

Parra, D. (2003). Manual de estrategias de enseñanzalaprendizaje. SENA. http://repositorio.minedu.gob.pe/handle/20.500.12799/4855

Pediatrics, A. A. (2018). Comportamiento normal de un niño ¿Cómo sé si el comportamiento de mi hijo es normal? Vida Familiar. https://revistas.uniandes.edu.co/doi/full/10.18175/vys9.2.2018.02

Peña, P., Sánchez, J., y Menjura, M. (2015). La convivencia en la escuela. Entre el deber ser y la realidad. Revista Latinoamericana de Estudios Educativos. https://www.redalyc.org/pdf/1341/134152136007.pdf

Pérez, I. (2016). Unidad De trabajo Social. Unidad de Trabajo Social: https://blogs.comillas.edu/uts/de-la-complejidad-a-la-inclusion/

Pérez, R. (2007). Educación, Ciudadanía y Convivencia. diversidad y sentido social de la educación. XIV Congreso Iberoamericano de Pedagogía, 243. file://C:/Users/USUARIO/ Downloads/Dialnet-

Piaget, J. (1996). Enfoque constructivista a la enseñanza de la convivencia. http://www.arje.bc.uc.edu.ve/arj19/art38.pdf 
RAE (2013). Diccionario de la Real Academia Española. Madrid: RAE. https://dle.rae.es/

RAE (2016). Estrategia. Real Academia Española -RAE. https://www.iesfuente.com/departamentos/latin_comun/lexico/ejer_ortogr/Ortografia_ rae.pdf

Ramírez, V. (2016). Democracia y Sociedad. Estudios Políticos. http://www.scielo.org.mx/pdf/ep/n38/0185-1616-ep-38-00143.pdf

Raven, E. (2015). Enfoque constructivista a la enseñanza de la convivencia. ARJÉ, 462. https://docplayer.es/91359821-Enfoque-constructivista-a-la-ensenanza-de-la-convivencia. html

Reimers, F., y Villegas, E. (2006). Sobre la calidad de la educación y su sentido democrático. PRELAC, 105. https://www.researchgate.net/publication/315011600_Sobre_la_Calidad_ de_la_Educacion_y_su_Sentido_Democratico

Reutert, G., y Castro, P. (2019). Teorías subjetivas de profesores acerca de su rol en la construcción de la convivencia escolar. Polis Revista Latinoamericana. https://scielo.conicyt.cl/scielo.php?pid=S0718-65682017000100321\&script=sci_abstract\&tlng $=\mathrm{p}$

Sánchez, C. (08 de febrero de 2019). Normas APA - 7ma (séptima) edición. Normas APA (7ma edición). https://normas-apa.org/

Sandoval, M. (2014). Convivencia y clima escolar: Claves de la gestión del conocimiento. Última Década N41, Proyecto Juventudes. https://scielo.conicyt.cl/scielo.php?pid=S0718-22362014000200007\&script=sci_arttext

Sierra, R. (2007). La estrategia pedagógica: Sus predictores de adecuación. Varona, 6. https://www.redalyc.org/pdf/3606/360635565004.pdf

Sikoyo, L. (2010). Contextual Challenges of Implementing Learner-centred Pedagogy: The Case of the Problem-solving Approach in Uganda. Cambridge Journal of Education. https://www.tandfonline.com/doi/abs/10.1080/0305764X.2010.509315

Torres, S., y Velandia, A. (2017). Infraestructura y equipamiento tecnológico en los doctorados consolidados de CONACyT: una mirada desde las politicas públicas y los académicos. Revista Iberoamericana de Educación Superior. http://www.scielo.org.mx/scielo.php?script=sci_arttext\&pid=S2007-28722017000100003 
Trujillo, D. (2018). Convivencia escolar y valores en estudiantes de grado octavo y noveno de la Institución Educativa Policarpa Salavarrieta del municipio de Quimbaya (Quindío). Universidad Nobert Wiener, 24. http://repositorio.uwiener.edu.pe/handle/123456789/1730

UNESCO (2005). Declaración de Dakar. UNESCO. https://repositorio.uam.es/handle/10486/667463

Valcárcel, N. (1998). Estrategia interdisciplinaria de superación para profesores de ciencias de la enseñanza media. Instituto José Varona.

Vygotsky, L. S. (1979). El desarrollo de los procesos psicológicos superiores. Buenos Aires: Grijalbo. http://sisep.minedu.gob.bo:8083/pdf/edu-reg/9_Lev,\%20Vygotzky_Desarro$110 \% 20$ procesos $\% 20$ psicologicos.pdf

Yamanija, C., Omura, D., y Barrientos, R. (2018). Disposición al aprendizaje y convivencia democrática en escuelas públicas del Perú. EDUCA UMCH, 16. 